NBER WORKING PAPER SERIES

\title{
CONTINGENT RESERVES MANAGEMENT: AN APPLIED FRAMEWORK
}

\author{
Ricardo J. Caballero \\ Stavros Panageas \\ Working Paper 10786 \\ http://www.nber.org/papers/w10786
}

\author{
NATIONAL BUREAU OF ECONOMIC RESEARCH \\ 1050 Massachusetts Avenue \\ Cambridge, MA 02138 \\ September 2004
}

Prepared for the Central Bank of Chile's conference on "External Vulnerability and Preventive Policies," Santiago Chile, August 2004. We are grateful to Andres Velasco and conference participants for their comments, and to Fernando Duarte and Jose Tessada for excellent research assistance. The views expressed herein are those of the author(s) and not necessarily those of the National Bureau of Economic Research.

(C)2004 by Ricardo J. Caballero and Stavros Panageas. All rights reserved. Short sections of text, not to exceed two paragraphs, may be quoted without explicit permission provided that full credit, including () notice, is given to the source. 
Contingent Reserves Management: An Applied Framework

Ricardo J. Caballero and Stavros Panageas

NBER Working Paper No. 10786

September 2004

JEL No. E2, E3, F3, F4, G0, C1

\section{ABSTRACT}

One of the most serious problems that a central bank in an emerging market economy can face, is the sudden reversal of capital inflows. Hoarding international reserves can be used to smooth the impact of such reversals, but these reserves are seldom sufficient and always expensive to hold. In this paper we argue that adding richer hedging instruments to the portfolios held by central banks can significantly improve the efficiency of the anti-sudden stop mechanism. We illustrate this point with a simple quantitative hedging model, where optimally used options and futures on the S\&P100's implied volatility index (VIX), increases the expected reserves available during sudden stops by as much as 40 percent.

Ricardo J. Caballero

Department of Economics

MIT

Room E52-252a

Cambridge, MA 02142-1347

and NBER

caball@mit.edu

Stavros Panageas

Department of Economics

MIT

Cambridge, MA 02142-1347 


\section{Introduction}

One of the most serious problems that a central bank in an emerging market economy can face, is the sudden reversal of capital inflows (sudden stops). Hoarding international reserves can be used to smooth the impact of such

reversals (see, e.g., Lee (2004)), but these reserves are seldom sufficient and always expensive to hold.

In Caballero and Panageas (2004) we derive and estimate a quantitative model to assess the (uncontingent) reserves management strategy typically followed by central banks. We conclude that this strategy is clearly inferior to one in which portfolios include assets that are correlated with sudden stops. As an illustration, we show that holding contracts on the S\&P100 implied volatility index (VIX) can yield a significant reduction in the average cost of sudden stops.

This result should not be surprising to those following the practices of hedge funds and other leading investors. Except for extremely high frequency events, which unfortunately sudden stops are not, institutional investors seldom immobilize large amounts of "cash" to insure against jumps in volatility and risk-aversion. The use of derivatives, and the creation of the VIX in particular, are designed precisely to satisfy hedging needs. Why should central banks, which aside from their monetary policy mandate, are the quintessential public risk management institutions, not adopt best-risk-management practices?

In this paper we revisit this point in the context of a simpler model designed to isolate the portfolio dimension of the reserves management problem. We estimate the key parameters of the model from the joint behavior of sudden stops and the VIX, which we then use to generate optimal portfolios. We show that in an ideal setting, where countries and investors can identify the jumps in the VIX and there exist call options on these jumps, an average emerging market economy may expect to face a sudden stop with up to 40 percent more reserves than when these options are not included in the central bank's portfolio.

The main reason behind this important gain is the close relation we identify between jumps in the VIX and sudden stops. We estimate that the 
probability of a sudden stop conditional on a jump in the VIX is about four times the probability of a sudden stop when there is no jump. Another dimension of the same finding but that speaks more directly of the hedging virtues of the VIX, is that while the probability of a jump in the VIX when there is no sudden stop in emerging markets is slightly above 30 percent, it rises to over 70 percent when a sudden stop takes place in that year.

Section 2 in the paper presents a simple static portfolio model for a central bank concerned with sudden stops. Section 3 presents the solution of the model under various assumptions on hedging opportunities. Section 4 discusses implementation issues. Section 5 quantifies the model. It starts by illustrating the behavior of the VIX and its coincidence with sudden stops in emerging markets (represented by nine economies: Argentina, Brazil, Chile, Indonesia, Korea, Malaysia, Mexico, Thailand and Turkey). It then estimates the different parameters of the model and reports the optimal portfolios for a range of relevant parameters. Section 6 documents the impact of the different hedging strategies on the availability of reserves during sudden stops. Section 7 concludes.

\section{Basic Framework}

We analyze the investment decisions of a central bank that seeks to minimize the real costs of a sudden stop of capital inflows. Our goal is to provide a simple model to isolate the portfolio problem associated with such an objective. We refer the reader to Caballero and Panageas (2004) for a dynamic framework that discusses the optimal path of reserves as well as the microeconomic frictions behind sudden stops. Here we simply take from that paper that when a sudden stop takes place, the country's ability to use its wealth for current consumption is significantly curtailed. The immediate implication of such a constraint is a sharp rise in the marginal value of an extra unit of reserves.

There are two dates in the model: date 0 , when portfolio decisions are made, and date 1 , when asset returns realize and a sudden stop may take 
place. We assume that a central bank has an objective of the form:

$$
\max _{R_{0}, \pi}-\frac{\alpha}{2} E\left[\left(R_{1}-K-1\{S S\} Z\right)^{2}\right]
$$

where $R_{1}$ denotes total reserves at date $1 . \quad K \geq 0$ is a "target" level of reserves at date 1 , which we take to be constant throughout, and captures reasons for holding reserves other than the (short run) fear of sudden stops we emphasize. Deviations from this target are costly: a shortfall from the target implies that the objectives of the central bank cannot be met adequately. Similarly, an excess level of reserves implies costs of accumulation (which among other things captures the difference between the borrowing and the lending rate, the slope of the yield curve, etc.). The term $1\{S S\} Z$ is composed of two terms. An indicator function $1\{S S\}$ that becomes 1 during a sudden stop (SS) and is 0 otherwise, and a constant $Z>0$ that controls the need for funds during the sudden stop. This constant captures the shift in the marginal utility of wealth that occurs once a sudden stop takes place. Hence, by construction of the optimization problem, a central bank desires to transfer reserves to sudden stop states. The program $((\mathrm{P}))$ is to be solved subject to:

$$
\begin{aligned}
& R_{0}=\pi P_{0}+B_{0} \\
& R_{1}=B_{1}+\pi P_{1}
\end{aligned}
$$

where $R_{0}$ is the initial level of reserves, $\pi$ is the amount of risky securities held by the central bank, $P_{0}$ is the price of such securities and $P_{1}$ is the (stochastic) payoff of these assets at $t=1$. $B_{0}$ is the amount of uncontingent bonds held by the central bank, whose interest rate we fix to 0 for simplicity, so that $B_{1}=B_{0}$, and

$$
R_{1}=R_{0}+\pi\left(P_{1}-P_{0}\right) .
$$

Replacing this expression in $((\mathrm{P}))$ and computing the first order conditions with respect to $R_{0}$ and $\pi$, yields:

$$
\begin{gathered}
R_{0}=K+\operatorname{Pr}(S S) Z \\
\pi=Z \frac{\operatorname{Cov}\left(1\{S S\}, P_{1}\right)}{\operatorname{Var}\left(P_{1}\right)}
\end{gathered}
$$


where we have removed Merton's portfolio term by assuming fair-risk-neutral pricing of the risky asset (an assumption we maintain throughout):

$$
E\left[P_{1}\right]=P_{0}
$$

There are three observations about this simple setup worth highlighting at this stage. First, the central bank has an aversion to over-accumulating reserves. If $Z=0$ and $K=0$, then $R_{0}=0$. Under these circumstances, the central bank achieves the maximum of the objective. Our main concern in this paper is with those reserves that are due to the possibility of a costly sudden stop, $Z>0$.

Second, the level of reserves invested at date $0, R_{0}$, is independent of the portfolio, $\pi$, or the properties of the risky asset. This is due to the "certainty-equivalence" property of the quadratic model. In fact, with more general preferences that exhibit a prudence motive, such as a the CRRA, an increase in hedging $(\pi)$ reduces the total amount of reserves held (see Caballero and Panageas, 2004).

Third, and most importantly, risky assets are not held if $P_{1}$ is uncorrelated with the sudden stop, $1\{S S\}$. Risky assets are only held to the extent that they succeed in creating attractive payoffs during sudden stops, i.e., as long as: ${ }^{1}$

$$
E\left[P_{1} \mid S S=1\right]>E\left[P_{1} \mid S S=0\right] .
$$

\section{From Conventional Reserves to Hedges}

Let us characterize the solution for a few cases of special interest. Our first base case model assumes away hedging completely, which is not far from what central banks do in practice. The second case is an Arrow-Debreu setup where contracts can be written contingent on the sudden stop. It captures the opposite extreme. The third is an intermediate case, that allows for "proxy" hedging through contracts that are correlated, but not perfectly, with sudden stops.

\footnotetext{
${ }^{1}$ Where we have re-normalized all potential assets to be held in positive amounts.
} 


\subsection{No Hedging}

Assume that we set $\pi=0$ in the base case model and drop the optimization with respect to $\pi$. Then obviously:

$$
B_{0}=R_{0}=K+\operatorname{Pr}(S S) Z \text {. }
$$

As one might expect, the possibility of a sudden stop induces the country to hold reserves beyond the "target" level $K$. This is probably one the main reasons why Chile, for example, holds four to five times as much reserves as Australia or Canada.

\subsection{Hedging with Arrow-Debreu Securities}

Taking the opposite extreme, assume that there exists an asset that pays:

$$
\left\{\begin{array}{l}
1 \text { if } S S=1 \\
0 \text { if } S S=0
\end{array}\right.
$$

In this case our assumption of fair-pricing implies

$$
P_{0}=\operatorname{Pr}(S S)
$$

It follows from (3) and the fact that in this case:

$$
\operatorname{Cov}\left(1\{S S\}, P_{1}\right)=\operatorname{Var}(1\{S S\})=\operatorname{Var}\left(P_{1}\right)
$$

that

$$
\pi=Z
$$

Replacing this expression in (1) and (2) we obtain:

$$
B_{0}=K+\operatorname{Pr}(S S)(Z-\pi)=K \text {. }
$$

Not surprisingly, with perfect Arrow Debreu securities (and fair pricing) the central bank will completely hedge away the sudden stop risk, so that:

$$
R_{1}-1\{S S\} Z=B_{0}=K
$$


Now let us express the portfolio of Arrow-Debreu securities as a proportion of total reserves:

$$
\phi=\frac{\pi P_{0}}{R_{0}}=\frac{\operatorname{Pr}(S S) Z}{K+\operatorname{Pr}(S S) Z} .
$$

In the interesting special case where $K=0$ (corresponding to the case where the country finds it optimal to hold no reserves in the absence of sudden stops) we have that:

$$
\phi=1 .
$$

That is, all resources are invested in Arrow Debreu securities.

\subsection{The intermediate case}

In reality, one neither observes Arrow Debreu securities nor does one observe contracts written contingent on the sudden stop (at least in an amount sufficient to insulate the country from it). There are good reasons for that: in practice, the sudden stop itself is unlikely to be fully contractible since its occurrence may depend on a country's actions and private information. Hence the practical relevance of the simple model proposed above rests critically on whether there are assets and trading strategies that could function as good substitutes for the idealized assets envisaged above. Let us develop a simple extension to the Arrow Debreu world above, by introducing an asset that pays 1 when an event that we call $J$ happens (corresponding to, e.g., a discrete drop in some asset price). We introduce the notation:

$$
\begin{aligned}
\psi^{h} & =\operatorname{Pr}(S S=1 \mid J=1) \\
\psi^{l} & =\operatorname{Pr}(S S=1 \mid J=0) \\
\eta & =\operatorname{Pr}(J=1) \\
\psi & =\operatorname{Pr}(S S=1)=\eta \psi^{h}+(1-\eta) \psi^{l}
\end{aligned}
$$

and assume that

$$
0 \leq \psi^{l} \leq \psi^{h} \leq 1 .
$$

In this case it is suboptimal for the country to invest all of its assets in risky securities, but in general is willing to invest some, provided that

$$
\psi^{h}>\psi^{l} \text {. }
$$


The new optimization problem yields:

$$
\begin{aligned}
\pi & =Z \frac{\left(\psi^{h}-\psi\right)}{(1-\eta)}=Z\left(\psi^{h}-\psi^{l}\right) \\
B_{0} & =K+\psi Z-\pi \eta .
\end{aligned}
$$

Notice that these formulas encompass the ones obtained previously. If $\psi^{h}=\psi^{l}$ then the two indicators are independent and thus $\pi=0$. However, as $\psi^{h} \rightarrow 1$ and $\psi^{l} \rightarrow 0$, then $\psi \rightarrow \eta$ and the country insures the sudden stop completely. Short of that, the central bank finds it optimal to do some of the hedging of sudden stops with uncontingent reserves: If $\psi^{h}<1$, there is a chance that the risky asset does not deliver during a sudden stop. And if $\psi^{l}>0$, the country pays for protection that does not need from the risky asset. $^{2}$

Note that as a percentage of total reserves, we have that the risky asset portfolio represents:

$$
\phi=\frac{\pi P_{0}}{R_{0}}=\frac{Z \eta}{R_{0}}\left(\psi^{h}-\psi^{l}\right) .
$$

This expression has a natural interpretation. Let $x \in[0,1]$ represent the share of reserves allocated to the prevention of sudden stops in the near future:

$$
x=\frac{\psi Z}{K+\psi Z} .
$$

Due to quadratic utility this number is independent of the hedging instruments (notice that the properties of $J$ do not influence this number). Then the optimal portfolio is:

$$
\phi=x \frac{\eta}{\psi}\left(\psi^{h}-\psi^{l}\right) .
$$

That is, the portfolio is composed of three terms: the first one is the fraction of reserves used for the prevention of sudden stops, $x .^{3}$ The second captures

\footnotetext{
${ }^{2}$ Note that with quadratic utility it suffices that $\psi^{l}=0$ and $\psi^{h}>0$ for the country to invest its entire portfolio in the risky asset (for $K=0$ ). The proximity of $\psi^{h}$ to one only determines how much hedging is achieved by this strategy.

${ }^{3}$ Note that our sense of prevention is different from that in Garcia and Soto (this volume) where a stock of reserves is needed to prevent runs on the country. Their concept is subsumed in our fixed $K$, although if runs are related to factors that tighten international financial markets, then this term also should be analyzed as an optimal portfolio decision.
} 
the relative frequency of jumps and sudden stops; as this rises the price of the insurance rises. The third one is the difference between the probability of a jump conditional on a sudden stop taking and not taking place. The latter term captures the risky asset's ability to transfer resources to the states where they are needed the most.

Dividing $\phi$ by $x$ isolates the share of the risky asset in the component of reserves used for hedging sudden stops. This is the concept we emphasize henceforth by setting $x=1$ ( or $K=0$ ).

\section{Implementation issues}

Let us now take the analysis one step closer to actual assets. For this purpose, we start by specifying a state variable, $s_{t}$, that is correlated with sudden stops but is not under the country's "control." Assume that $s_{t}$ evolves according to the (discretized) stochastic differential equation:

$$
s_{t+1}-s_{t}=\mu\left(s_{t}\right) \Delta t+\sigma N(0,1) \sqrt{\Delta t}+\varepsilon d J_{1}
$$

where $\mu\left(s_{t}\right)$ is the drift (the mean appreciation rate of the state variable), and $\sigma$ is the volatility. The most interesting part of this expression is the jump process $d J_{1}$, which is zero except at date 1 , when it takes the value one with probability $\eta$ and zero otherwise, in perfect analogy to the setup in section 3.3. We let $\varepsilon$ be a random variable normally distributed, with mean $\mu_{\varepsilon}>0$ and standard deviation $\sigma_{\varepsilon}$.

\subsection{Call Options}

Given the above framework, we consider the following thought experiment: Is there a simple strategy that can "create" an asset of the sort envisaged in section 3.3 by writing contracts contingent on $s_{t}$ ? The answer is yes. To see this, take the continuous time limit of (8) and consider a contract with an investment bank or insurer in which the central bank pays an amount $\kappa d t$ in exchange for each dollar received if $s_{t}$ exhibits a jump at $t=1$. In continuous time such a contract is well defined. In reality, one can approximate it by signing a sequence of appropriate (sufficiently out of the money) "digital" 
options (furthermore, such options can be well approximated by regular puts and calls) which cost $\eta d t$ per unit of time. The cost of such a position over the full period is:

$$
\int_{0}^{1} \eta d t=\eta
$$

and the payoff is one if a jump happens at $t=1$, and zero otherwise. Notice that this strategy is also feasible if one extends the model to the case where a jump in $s_{t}$ can happen at any time $\tau$ as in Caballero and Panageas (2004). In fact, this is the process we estimate in the empirical section.

In conclusion, this sequence of short term "digital" options is for all practical purposes identical to the contract described in section $3.3{ }^{4}$

\subsection{Futures contracts}

Consider now simple futures contracts. If investors are risk neutral with respect to $s_{t}$ risk, a futures contract on $s_{t}$ with maturity at $t=1$ can be entered into at a forward "price" of:

$$
P_{0}=E\left[s_{1}\right]
$$

with return:

$$
s_{1}-E\left[s_{1} \mid s_{0}\right] .
$$

The expected payoff of such a position at $t=1$ is approximately: ${ }^{5}$

$$
\widetilde{v} \sim N\left(-\eta \mu_{\varepsilon}, \sigma\right)+1\{J\} N\left(\mu_{\varepsilon}, \sigma_{\varepsilon}\right)
$$

where $\{J\}$ corresponds to an indicator function that takes value one when a jump in the state variable takes place, and zero otherwise.

It is important to note that futures have a price of zero. However, in order to keep the analysis comparable with the results obtained in section 3.3 we consider a slight variation of a futures contract and assume that the

\footnotetext{
${ }^{4}$ We refer the reader to Caballero and Panageas (2004) for a more extensive discussion of these issues.

${ }^{5}$ To make the argument in this section exact one needs a continuous time model.
} 
country must pay $\eta \mu_{\varepsilon}$ upfront for every contract that it enters in exchange for a payoff of :

$$
v \sim N(0, \sigma)+1\{J\} N\left(\mu_{\varepsilon}, \sigma_{\varepsilon}\right) .
$$

Hence, the solution to the problem $((\mathrm{P}))$ in this case is

$$
\begin{aligned}
\pi \mu_{\varepsilon} & =Z \frac{\psi^{h}-\psi}{\left(\frac{\sigma^{2}+\eta \sigma_{\varepsilon}^{2}}{\eta \mu_{\varepsilon}^{2}}+1-\eta\right)}=Z \frac{1-\eta}{\left(\frac{\sigma^{2}+\eta \sigma_{\varepsilon}^{2}}{\eta \mu_{\varepsilon}^{2}}+1-\eta\right)}\left(\psi^{h}-\psi^{l}\right) \\
B_{0} & =K+\psi Z-\pi \eta \mu_{\varepsilon}
\end{aligned}
$$

There are several observations about $\pi$ that are worth highlighting. First, we can set $\mu_{\varepsilon}=1$ without loss of generality, since the dollar amount invested in the risky asset is $\pi \mu_{\varepsilon}$ at a price of $\eta$ per dollar invested. Moreover, observe that the right hand side depends only on the ratios $\frac{\sigma}{\mu_{\varepsilon}}, \frac{\sigma_{\varepsilon}}{\mu_{\varepsilon}}$. Hence from now on let us set $\mu_{\varepsilon}=1$ and denote $\widetilde{\sigma}=\frac{\sigma}{\mu_{\varepsilon}}$ and similarly for $\widetilde{\sigma}_{\varepsilon}, \tilde{\pi}$. Thus, in dollar amounts we have:

$$
\widetilde{\pi}=Z\left(\psi^{h}-\psi^{l}\right) \frac{1-\eta}{\frac{\sigma^{2}}{\eta}+\widetilde{\sigma}_{\varepsilon}^{2}+1-\eta}
$$

Comparing (9) to (6) shows that the amount invested in risky assets declines when going from digital options on the jump to the simple futures (the denominator is larger in (9)). The ratio between the two portfolios is:

$$
\frac{1-\eta}{\frac{\sigma^{2}}{\eta}+\widetilde{\sigma}_{\varepsilon}^{2}+1-\eta}<1
$$

which declines as $\widetilde{\sigma}, \widetilde{\sigma}_{\varepsilon}$ increase. This is intuitive: The more noise there is in the hedging opportunities, the less appealing they become to a risk averse central bank. Note that the portfolio $\phi$ also is attenuated by the ratio in (10).

To summarize, we have that in order to justify adding a risky asset to the central bank's holdings, sudden stops must be severe, and the risky asset must be sufficiently correlated with such events. On the other hand, it is important to emphasize that neither causality nor predictability of sudden stops and returns are part of the argument for a positive $\pi$. 


\section{Quantitative Assessment}

The theoretical argument for hedging is difficult to argue with. The relevant question is then an empirical one: Are there global financial instruments and indices that offer good enough hedging opportunities against sudden stops? Obviously, the answer to this question is to a large extent country-specific, as not all emerging market economies are exposed to the same sources of fragility. Our goal in this section is more modest but also more robust: Rather than performing a collection of cases studies, we show that there is at least one global asset that has significant correlation with emerging market crises. More importantly, absent better country-specific alternatives, this global asset should constitute a significant share of these countries portfolios.

\subsection{The basics: Sudden Stops and Jumps}

We study a group of nine emerging market economies open to international capital markets during the 1990s for which we have complete data: ${ }^{6}$ Argentina, Brazil, Chile, Indonesia, Korea, Malaysia, Mexico, Thailand and Turkey. These economies are representative of what is often referred to as "emerging market economies." Our main exclusion is the group of Eastern and Central European economies, who became significant participants in international capital markets during the second half of the 1990s, but faced economic problems of a somewhat different nature during much of our sample.

The first point to highlight is the well known fact that there is significant comovement in private capital flows to these economies. Figure 1 splits into two panels the paths of the change in capital flows - more precisely, the difference between contiguous four-quarter-moving averages of quarterly capital flows - to each of these economies from 1992 to 2002. The shaded areas mark the periods corresponding to the systemic Tequila crisis, Asian crisis, and Russian crisis, and the sequence of somewhat less systemic TurkishArgentinean-Brazilean crises. It is apparent from this figure that there are significant correlations across these flows, especially within regions. Turkey

\footnotetext{
${ }^{6}$ The exception is Malaysia, for which we do not have quarterly capital flows.
} 
is somewhere in between the two regions. These comovements are encouraging, as they indicate the possibility of finding global factors correlated with sudden stops.
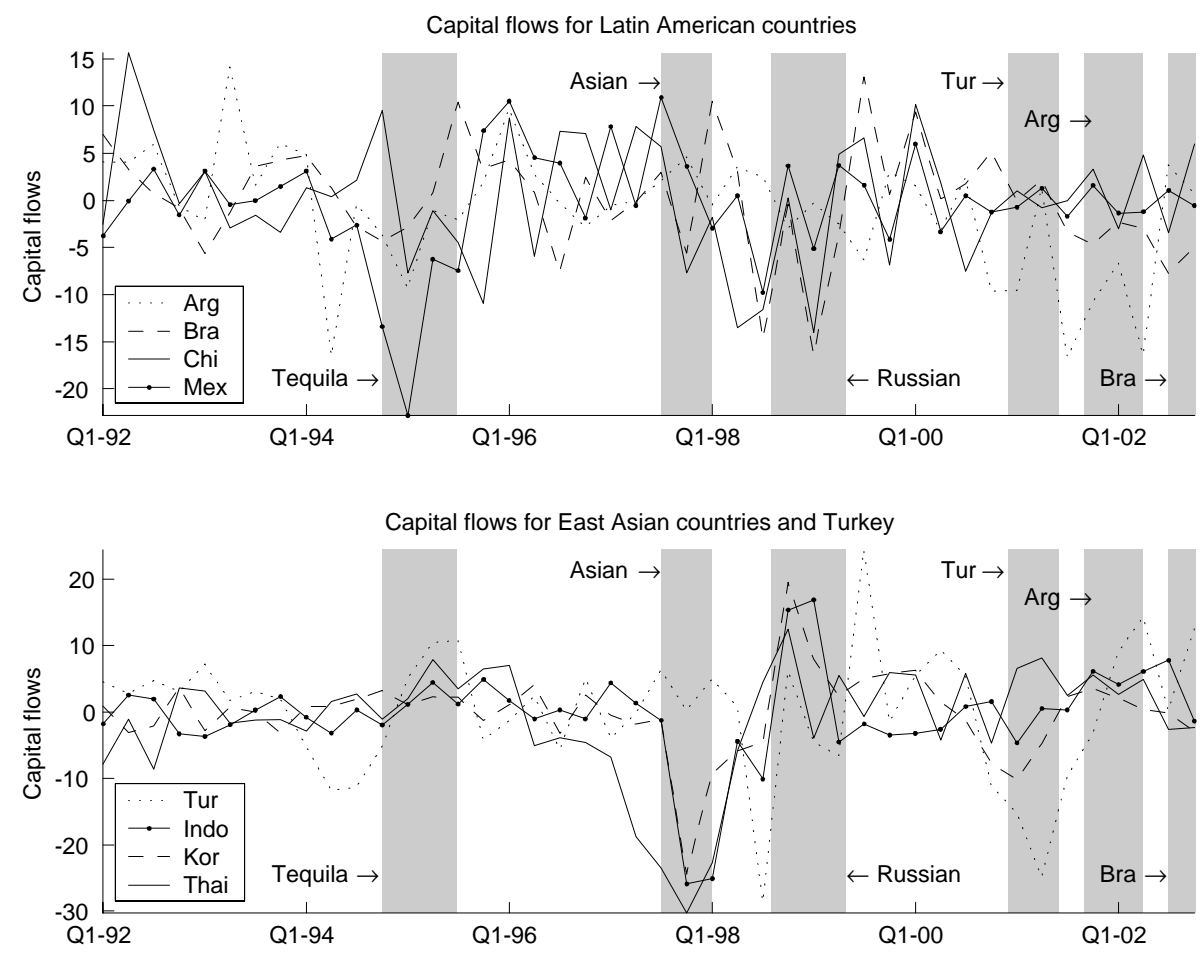

Figure 1: Capital flows for various countries. The figure depicts the difference between 4 quarter averages of capital flows and the same quantity one year before. The shaded areas show times of major crises.

The second, and main point, is that indeed there are clearly identifiable global factors - in fact, traded factors - correlated with emerging market sudden stops. The key in finding such factors is to note that these episodes are generally understood as times when investors are reluctant to participate in risky markets. The VIX precisely captures this reluctance and is available in the US since 1986. This is an index of the "implied volatilities" from puts and calls (typically 8) on the S\&P 100. (Implied volatilities are 


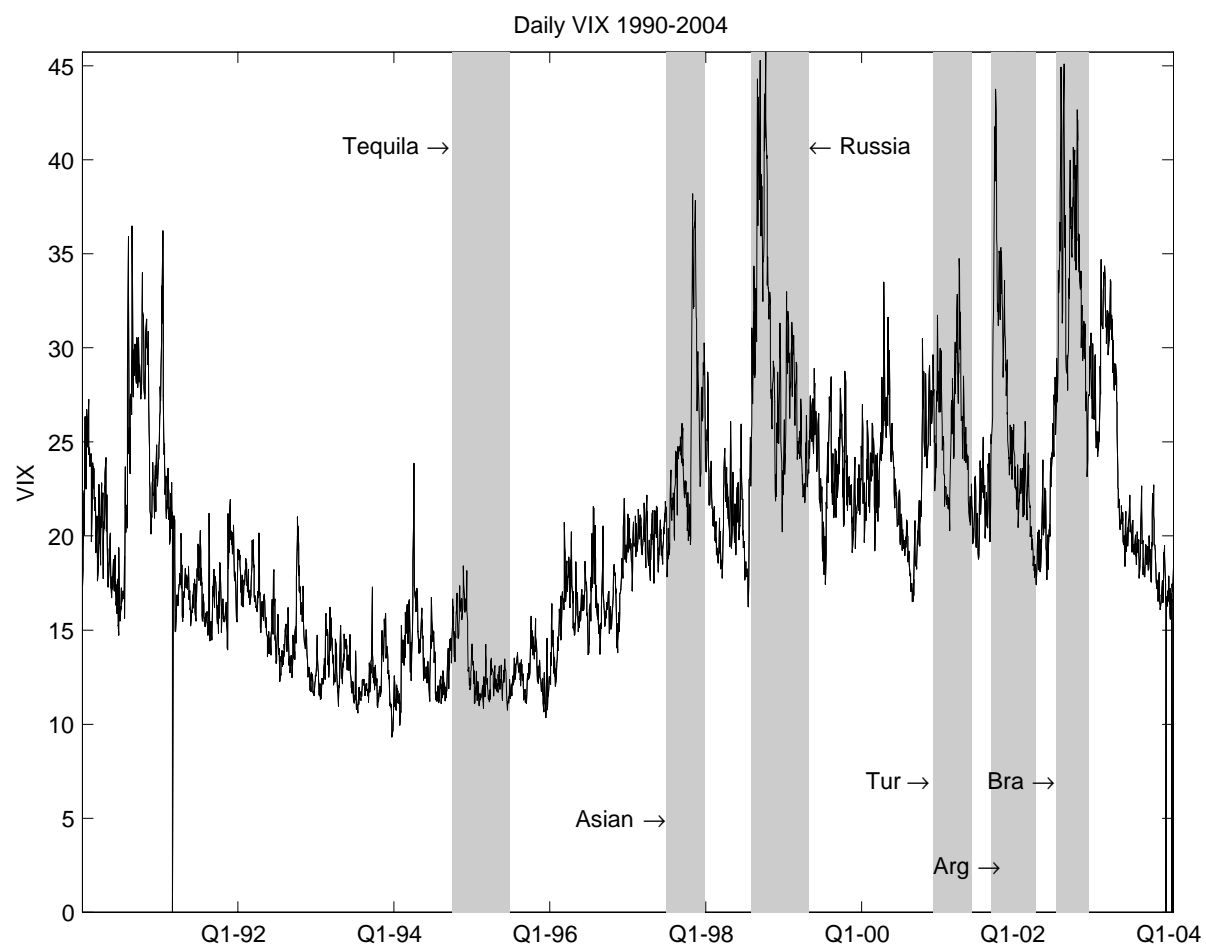

Figure 2: Daily VIX series. The gray areas depict the periods of major crises.

determined by using the Black and Scholes (1973) formula to determine the level of volatility that would be compatible with the observed prices of puts and calls). Figure 2 reproduces the shaded areas for sudden stops regions described in the previous graph, and plots the daily VIX. It is apparent in this figure that some of the largest "jumps" in the VIX occur precisely during sudden stops. In fact, the only systemic sudden stop that does not coincide with a jump is the Tequila crisis, where there was a rise in the VIX but not large enough to count as a distinct jump.

In the next section we document formally the joint behavior of sudden stops and jumps in the VIX. But before doing so, it is instructive to explore in more detail the behavior of the VIX during the two largest systemic crises of the 1990s (the Asian and Russian crises). The top panel in Figure 3 plots the 
path of the VIX during the last two weeks of October 1997 (the onset of the Asian Crisis), while the bottom panel does the same for August-September 1998, which corresponds to the peak of the Russian/LTCM crisis. In these events the VIX reaches levels above 30 and 45 percent, respectively, which are close to the maximum levels of the index. In a matter of days, the VIX doubled.
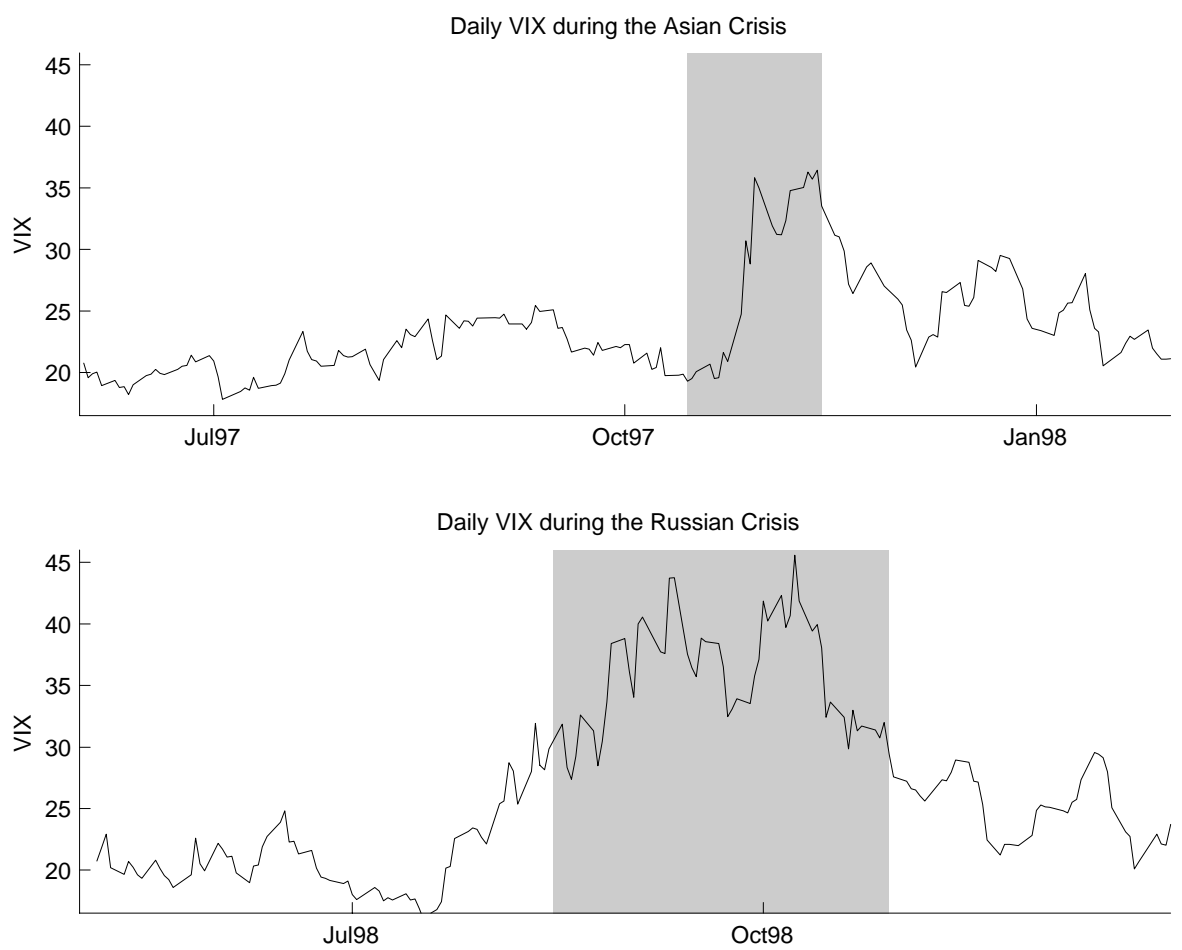

Figure 3: Plots of the VIX in the weeks surrounding major crises in international financial markets.

Finally, Figure 4 reinforces the message of high correlation by plotting the path of the VIX together with the EMBI for three of the most fragile economies in emerging markets during recent years: Argentina, Brazil and Turkey. Again, the dips in the corresponding EMBIs as the VIX experiences 
sharp rises are apparent. ${ }^{7}$ A variable that is primarily meant to capture the "feelings" of investors in US equity markets, happens to be highly correlated with the fortunes of emerging market economies. This highlights another important aspect of our methodology, according to which the only requirement for a variable like the VIX to be useful in hedging, is that there be a change in the conditional probability of having a crisis in emerging markets too. This is not a statement about causation, but about correlation.

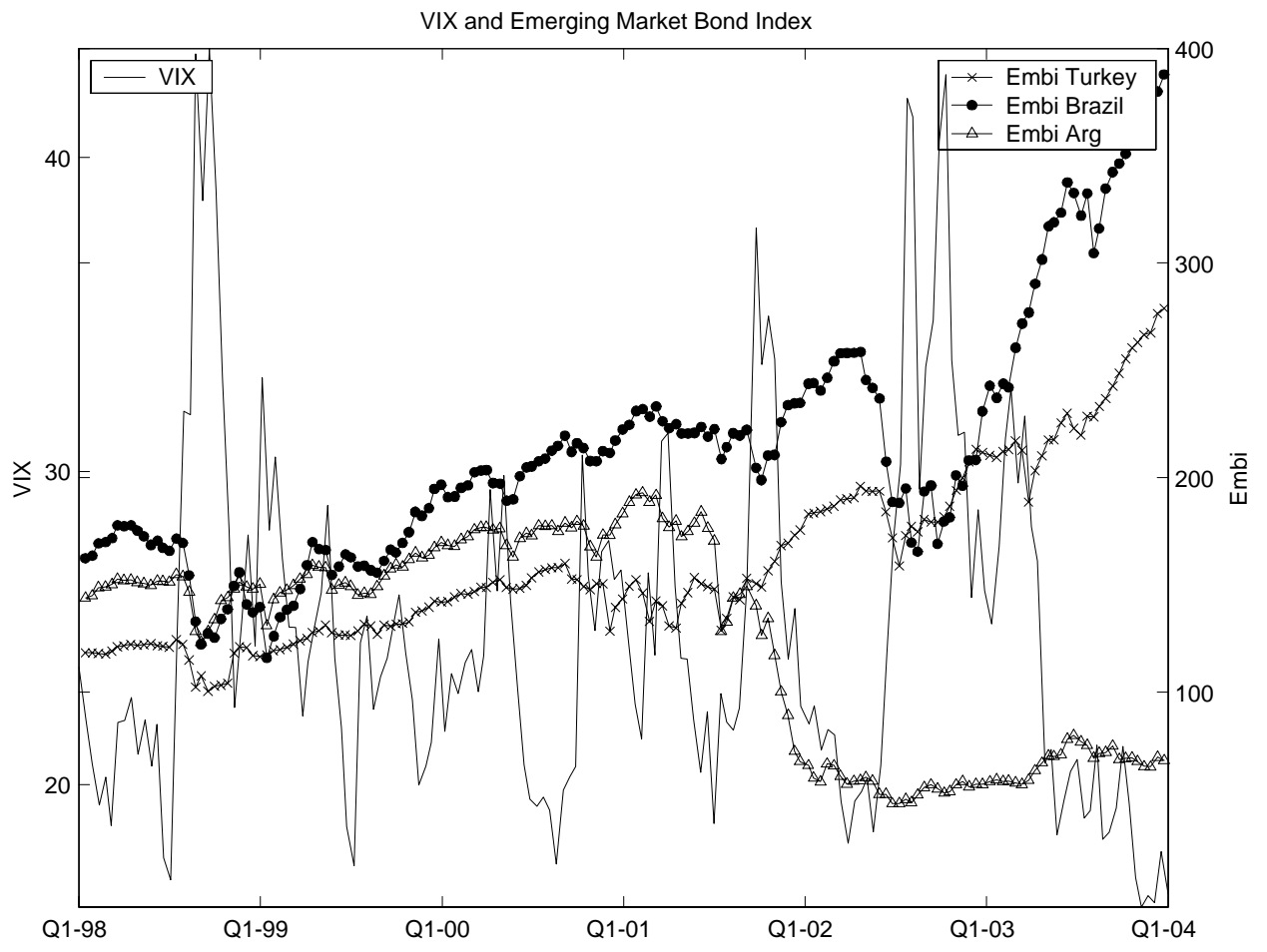

Figure 4: The VIX and the EMBI for Argentina, Brazil and Turkey.

In concluding this section we stress that our claim is not that domestic factors do not play a paramount role in crises. Quite the contrary, our choice of Argentina, Brazil and Turkey in the previous figure is precisely because

\footnotetext{
${ }^{7}$ The EMBI data are from Datastream. Note that the Argentine (permanent) crash also coincides with a spike in the VIX.
} 
their own domestic weaknesses make them more responsive to global factors. Importantly, this compounding effect often raises rather than dampens the need for hedging global risk factors.

\subsection{Quantification}

We now turn into a structural analysis of the correlations highlighted above.

\subsubsection{Estimation of the VIX process}

To operationalize the model of the previous section take the $\log$ (VIX) to be the state variable $s_{t}$, which follows the continuous time process:

$$
d s_{t}=-\theta\left(\log \left(s_{t}\right)-y\right) d t+\sigma d B_{t}+\varepsilon d J_{t}
$$

This is the continuous time limit of (8), with the modification that jumps can happen at any point in time. 8 The functional form $\mu\left(s_{t}\right)=-\theta\left(\log \left(s_{t}\right)-y\right)$, corresponds to an $\mathrm{AR}(1)$ process in discrete time for the $\log \left(s_{t}\right)$. Thus, we start by estimating an $\mathrm{AR}(1)$ process for $\log (V I X)$ with monthly data and focus on the residuals, $v$, which are distributed (for small $\Delta t$ ) roughly as

$$
v \sim(1-p) N\left(-\eta \mu_{\varepsilon} \Delta t, \sigma \sqrt{\Delta t}\right)+p N\left(\mu_{\varepsilon}, \sigma_{\varepsilon}\right)
$$

Given the very few observations with jumps, we identify these directly by inspection; this process fixes $\eta=0.417$ and hence $p=1-e^{-\eta \Delta t}$. The rest of the parameters are estimated by maximum likelihood of the mixing of two normal distributions. Table 1 reports the results.

\begin{tabular}{lcccc}
\hline \hline & $\eta$ & $\mu_{\epsilon}$ & $\sigma$ & $\sigma_{\epsilon}$ \\
\hline Estimate & 0.417 & 0.356 & 0.353 & 0.047 \\
\hline \hline
\end{tabular}

Table 1: Estimated parameters for monthly VIX data

\footnotetext{
${ }^{8}$ This is not a serious departure if the "horizon" in the decision model is understood to be one year and the probability of more than 1 jump taking place in a single year is small.
} 


\subsubsection{The Likelihood of Sudden Stops}

The results in section 5.2.1 suggest the presence of five jumps in the VIX in our sample: The gulf war, the Asian crisis, the Russian crisis, 9/11 and the simultaneous crisis of Turkey/Brazil, and the corporate scandals in the US. Conditional on these jumps, we calculate the probability that a country experiences a sudden stop. We identify an observation as a sudden stop based on a mixture of information on capital flows reversals and reserves losses (see Caballero and Panageas 2004). Mainly, for an observation to count as a sudden stop capital flows must decline by at least 5 percent of GDP with respect to the flows in the previous two years, and reserves must be declining. This procedure yields estimates of $\psi^{h}$ and $\psi^{l}$ for each country. We then estimate $\psi$ from the relation:

$$
\psi=\eta \psi^{h}+(1-\eta) \psi^{l} .
$$

The results are reported in Table 2. Note, however, that the countryspecific estimates are highly imprecise as they correspond to the product of binary variables with very few transitions in each case. ${ }^{9}$ For this reason, we pool the observations, which yields the result in the average row. We also report results for two sub-categories: High-risk economies (Argentina, Brazil, and Turkey) and for the East-Asian economies. The former group is composed of those economies that have the highest estimated likelihood of a sudden stop in the sample.

The pooled estimate indicates that an average emerging market economy is more than four times more likely to experience a crisis at a time when the VIX has jumped than when it has not. Again, this is not a statement of causation but of correlation. At times when the VIX spikes, the average emerging market economy has a 41 percent chance of experiencing a sudden stop. This chance drops to 11 percent when the VIX is tranquil.

\footnotetext{
${ }^{9}$ The case of Mexico is particularly revealing. The estimate of $\psi^{h}=0$ misses the fact that while Mexico did not experience a very significant capital flow reversal during the Russian/LTCM/Brazilean turmoil, its stock market declined very sharply, reflecting that it experienced significant pressure at the time, but adjusted primarily via prices instead of quantities. Chile and the East Asian countries have a $\psi^{l}=0$ because we identify only a single SS for each of them and we observe a jump in the VIX during the same period.
} 


\begin{tabular}{lccc}
\hline \hline & $\psi$ & $\psi^{h}$ & $\psi^{l}$ \\
\hline Argentina & 0.42 & 0.80 & 0.14 \\
Brasil & 0.42 & 0.60 & 0.29 \\
Chile & 0.17 & 0.40 & 0.00 \\
Mexico & 0.17 & 0.00 & 0.29 \\
Indonesia & 0.17 & 0.40 & 0.00 \\
Korea & 0.08 & 0.20 & 0.00 \\
Malaysia & 0.25 & 0.40 & 0.14 \\
Thailand & 0.17 & 0.40 & 0.00 \\
Turkey & 0.33 & 0.60 & 0.14 \\
\hline Average & $\mathbf{0 . 2 4}$ & $\mathbf{0 . 4 1}$ & $\mathbf{0 . 1 1}$ \\
High-risk Countries & $\mathbf{0 . 3 9}$ & $\mathbf{0 . 6 7}$ & $\mathbf{0 . 1 9}$ \\
East Asia & $\mathbf{0 . 1 7}$ & $\mathbf{0 . 3 5}$ & $\mathbf{0 . 0 4}$ \\
\hline \hline
\end{tabular}

Table 2: Estimates for $\psi, \psi^{h}$ and $\psi^{l}$. $\psi^{h}$ is estimated as the number of years when we observe a joint jump in the VIX and a SS in the country divided by the number of jumps in the VIX. Symmetrically, $\psi^{l}$ is the ratio of the number of years in SS when there was no jump divided in the total number of years without a jump. In order to determine whether a SS and a jump coincide we allow for a 2-quarter window around the date when we identify the jump, because jumps are identified at a higher frequency than SS. With the estimates for $\eta, \psi^{l}$ and $\psi^{h}$ in hand we obtain the estimate for $\psi$ presented in the first column of the table. 


\begin{tabular}{lcc}
\hline \hline & $\phi$ (Options) & $\phi$ (Futures) \\
\hline Argentina & 0.66 & 0.13 \\
Brasil & 0.31 & 0.06 \\
Chile & 1.00 & 0.20 \\
Mexico & 0.00 & 0.00 \\
Indonesia & 1.00 & 0.20 \\
Korea & 1.00 & 0.20 \\
Malaysia & 0.43 & 0.08 \\
Thailand & 1.00 & 0.20 \\
Turkey & 0.57 & 0.11 \\
\hline Average & $\mathbf{0 . 5 3}$ & $\mathbf{0 . 1 0}$ \\
High-risk Countries & $\mathbf{0 . 5 1}$ & $\mathbf{0 . 1 0}$ \\
East Asia & $\mathbf{0 . 7 9}$ & $\mathbf{0 . 1 6}$ \\
\hline \hline
\end{tabular}

Table 3: Representative portfolios for options and futures.

\subsection{Representative VIX-portfolios and Reserves Gains}

With these numbers in hand we are able to operationalize formulas (7) and (10) to estimate the portfolios implied by the model. Again, country specific numbers are very imprecise and attention should be placed on the pooled results. Table 3 reports the portfolios. The values of $\phi$ are large. The futures contracts show shares of risky assets of 10 percent or higher for the different groupings, despite the large amount of noise in the VIX. When the noise is removed and the call-options strategy is followed, the shares rise to above 50 percent in all cases, and to near 80 percent for the Asian economies. The reason for the high share for East Asian economies is worth highlighting: in the sample they experience crises mostly when these are systemic (again, this is not a causal statement); this is in contrast with the high risk economies, which also experience idiosyncratic crises. ${ }^{10}$

These are dramatically different portfolios from those normally held by central banks in emerging markets. Finding out why seems imperative: Is it

\footnotetext{
${ }^{10}$ Note also that the difference between the optimal precautionary behavior of a high risk and an average economy is not only reflected in the different $\phi$ s but also on the level of reserves held. Recall that $R_{0}=K+\psi Z$.
} 
the lack of liquidity of the potential markets, domestic political constraints, or simply institutional herding?

\section{The Benefits}

Our reduced form portfolio model is not well suited for a thorough "welfare" comparison. Thus, in assessing the benefits of the hedging strategy, we rather focus on statistics that are robust across preferences and other hard to quantify details. In particular, we report the expected gains conditional on a sudden stop taking place. We illustrate this for the call-options scenario.

The first step in computing this statistic is to estimate the likelihood of a jump given that the country has experienced a sudden stop. Using Bayes' rule, we have that:

$$
\operatorname{Pr}(J=1 \mid S S=1)=\psi^{h} \frac{\eta}{\psi} .
$$

Column 1 in Table 4 reports the estimates. It is around 70 percent for an average emerging market economy and close to 90 percent for the relatively stable East Asian economies. This is important. The VIX jumps with a high likelihood at times when the countries need it to do so.

The rate of return of the "call" strategy is:

$$
\left\{\begin{array}{c}
1 / \eta-1 \text { if } J=1 \\
-1 \text { if } J=0 .
\end{array}\right.
$$

Hence the expected gain in reserves conditional on entering a sudden stop is:

$$
\phi\left(\psi^{h} \frac{\eta}{\psi}(1 / \eta-1)-\left(1-\psi^{h} \frac{\eta}{\psi}\right)\right) .
$$

Column 2 in Table 4 reports the results. For an average economy, the expected gain is around 40 percent. That is, an average economy following the strategy described here can expect a 40 percent rise in its reserves upon entering into a sudden stop. ${ }^{11}$ This is a significant number, which exceeds

\footnotetext{
${ }^{11}$ Of course, the counterpart of this expected gain during sudden stops is that the economy may expect to lose 13 percent of its reserves when there is no sudden stop.
} 


\begin{tabular}{lcc}
\hline \hline Country & $\operatorname{Pr}(J=1 \mid S S=1)$ & Expected Gain (options) \\
\hline Argentina & 0.80 & 0.60 \\
Brasil & 0.60 & 0.14 \\
Chile & 1.00 & 1.40 \\
Mexico & 0.00 & 0.00 \\
Indonesia & 1.00 & 1.40 \\
Korea & 1.00 & 1.40 \\
Malaysia & 0.67 & 0.26 \\
Thailand & 1.00 & 1.40 \\
Turkey & 0.75 & 0.46 \\
\hline Average & $\mathbf{0 . 7 2}$ & $\mathbf{0 . 3 9}$ \\
High-risk Countries & $\mathbf{0 . 7 1}$ & $\mathbf{0 . 3 6}$ \\
East Asia & $\mathbf{0 . 8 8}$ & $\mathbf{0 . 8 6}$ \\
\hline \hline
\end{tabular}

Table 4: Revised probabilities and Expected Gains when following the options strategy.

the actual reserves losses of many of these economies during their respective sudden stops.

Of course, there are many caveats that can be raised and that are likely to reduce these large numbers. For example, in a dynamic model the central bank might find it optimal to hold a level of reserves above a certain minimum in all contingencies, even in "good" states. In the present model this is just equivalent to assuming that the central bank targets a non-zero level of reserves, i.e. $K>0$, which implies $x<1$. As we know from expression (7), the portfolio of risky assets is scaled down proportionally with $x$. Alternatively, one could imagine a situation where a central bank wishes under no circumstances to lose more than $c$ percent of its reserves, in which case the optimal portfolio would become:

$$
\min \{c, \phi\} .
$$

All these caveats notwithstanding, we feel that the above calculations make a simple point: no matter which assumptions we make about preferences and constraints, the driving force behind our results is the strong 
correlation between the VIX index and the incidence of sudden stops. The simple quadratic framework that we propose is particularly well suited to make this separation between preferences (which solely affect $x$ ) and correlation explicit. Even though a more elaborate model like that in Caballero and Panageas (2004) is required in order to have a satisfactory theory for $x$, the effects that come from the strong correlations are independent of the specifics of the model.

\section{$7 \quad$ Final Remarks}

We shall start our conclusion with a disclaimer. The portfolios we illustrate for the emerging market economies we study, and the emphasis on the VIX, are neither country-specific nor instrument-specific recommendations. Our goal is simply to illustrate the potential benefits of enriching the portfolio options for central banks and, most importantly, of searching for assets and indices that are global in nature but correlated with capital flow reversals.

Within this limited goal, our results are promising: the expected gains in reserves during sudden stops can be significant (slightly less than 40 percent of reserves for an average country). This is noteworthy, considering that we are only considering a single risky asset which is not optimized to capture the risks faced by emerging market economies.

The latter point raises an issue of international financial architecture: The VIX is useful because it is correlated with implied volatilities and risks in emerging markets but it also captures problems that are US-specific. Ideally, one would want an index that weights differently US-events that are likely to have world-wide systemic effects from those that do not. It should be relatively easy to construct implied volatility indices that isolate the former factors and still preserve the country-exogeneity properties of the VIX. Constructing such indices is important to create benchmarks and develop liquid hedging markets for economies exposed to capital flow volatility.

An issue that we avoided entirely is the incentive effects that a modified central banks' policy of hedging external shocks may have on the private sector. This is an important concern, as the private sector may undo some of the external insurance in anticipation of a the central bank's intervention. This 
is a complex issue that probably requires coordination of the hedging policy with monetary and regulatory policies (see Caballero and Krishnamurthy 2003). However, even in the absence of these complementary policies, perverse incentive effects are unlikely to be strong enough to fully offset the justification for more aggressive hedging practices. After all, current reserve policies also suffer from these problems and are justified on the grounds that many in the private sector are simply not forward-looking enough to hedge aggregate risks in sufficient amount.

Moreover, if such practices were to be adopted collectively, soon we would observe the emergence of new implied volatility indices that better match the needs of emerging market economies. The welfare improvement from such enhancements could be very significant and therefore may justify a coordination role by the IFIs and central banks around the world. In fact, such coordination may be a necessity, if we are to limit the potential political costs from hedging losses.

To conclude, we reiterate that our emphasis on external sources of capital flow volatility does not seek to shift the blame for much of capital flow volatility away from the countries themselves. Our goal is simply to show that there is a hedgable component and that this component is significant. Moreover, there is an important interaction between the issue we highlight here and the domestic sources of external fragility: Weak countries are more likely to be hit by global turmoil, and hence should put an even bigger effort in hedging these global shocks. 


\section{References}

[1] Caballero, R.J. and A. Krishnamurthy, "Inflation Targeting and Sudden Stops," MIT Mimeo, 2003.

[2] Caballero, R.J. and S. Panageas, "Hedging Sudden Stops and Precautionary Recessions: A Quantitative Approach," MIT Mimeo, 2003.

[3] Caballero, R.J. and S. Panageas, "Insurance and Reserves Management in a Model of Sudden Stops," MIT Mimeo, 2004.

[4] Garcia, P. and C. Soto, "Large Hoarding of International Reserves: Are they worth it?", in this volume, 2004.

[5] Jaewoo Lee, "Insurance Value of International Reserves," IMF mimeo, August 2004. 